\title{
ON THE RISK OF CRACKING IN CLAY DRYING
}

\author{
$\underline{\text { F. Augier }}^{1}$, W. J. Coumans ${ }^{1}$, A. Hugget ${ }^{1}$, E. F. Kaasschieter ${ }^{2}$ \\ Eindhoven University of Technology, \\ ${ }^{1}$ Laboratory of Separation Technology and Transport Phenomena \\ ${ }^{2}$ Department of Mathematics and Computing Science \\ P.O. Box 513, 5600 MB Eindhoven, The Netherlands \\ E-mail: f.f.augier@tue.nl
}

Keywords: clay drying, elasticity, cracking criterion

\begin{abstract}
Based on assumptions related to porous media, the governing equations of mass transfer and static equilibrium are presented. The mechanical stresses generated by the drying strains are expressed according to the linear-elastic model. The von Mises cracking criterion is introduced in order to locate the area where risk for cracking occurs.

The model is applied to the drying of Kaolin clay. Moisture and solid displacements results as well as evolutions of the criterion are exposed. Danger for cracking is highest at the beginning of the drying, since the yield stress is low. The criterion reaches its peak value during the first hour and at a particular point, located on the surface, exactly in-between two corners.

Moisture evolution has been measured by means of Nuclear Magnetic Resonance imaging, during the drying of a piece of Kaolin clay. The diffusion coefficient is evaluated from these experimental results. Finally, the model is used to reproduce them.
\end{abstract}

\section{INTRODUCTION}

When the drying process takes place with shrinkage, gradients of moisture content and solid displacement in the material will lead to drying-induced stresses. Controlling these stresses is important since they can lead to undesired deformations and/or cracks affecting the product quality.

Mixture of clay and water is considered in a first assumption as perfectly elastic, because of the lack of data about rheological properties on clay material. A model coupling mass transfer with stress balance, can permit to calculate the amount of stresses, according to several physical properties determined experimentally. The Huber-von Mises criterion is used to anticipate crack appearance.

After the presentation of the mathematical model, a numerical formulation using the finite element method is developed and the results applied to clay brick drying are exposed and analysed. Cracking criterion evolutions allow to understand cracking occurrence. Finally a validation of the diffusion model is given, by means of a comparison between simulated and experimental data. 


\section{THE MATHEMATICAL MODEL}

\section{Assumptions}

- clay is a solid porous medium (density $\rho_{\mathrm{s}}$ ) which can hold moisture (density $\rho_{\mathrm{m}}$ )

- moisture may be present in clay indifferently as vapor, free or bound liquid

- temperature of clay form is uniform and constant

- the effect of gravity is neglected

- clay material is isotropic

- mixture of clay and moisture shows a perfectly elastic behavior within the moisture range

$[0-0.4 \mathrm{~g} / \mathrm{g}]$. This choice is a limiting assumption.

\section{The diffusion model}

As a shrinking medium, clay shows a movement of its solid skeleton while water moves through it. Using the moisture content $\mathrm{M}$ ( $\mathrm{kg}$ water $/ \mathrm{kg}$ dry solid) as state variable, moisture transport during clay drying is described by equations (1) where $\mathrm{v}_{\mathrm{s}}(\mathrm{m} / \mathrm{s})$ denotes the solid velocity, $\mathrm{D}\left(\mathrm{m}^{2} / \mathrm{s}\right)$ the diffusion coefficient of water in clay and $\mathrm{J}\left(\mathrm{kg} / \mathrm{m}^{2} . \mathrm{s}\right)$ the moisture flux at the boundary surfaces exposed to drying conditions (Hugget, 1999; Wigmans, 1994; Ketelaars, 1992):

$$
\begin{cases}\frac{\partial \mathrm{M}}{\partial \mathrm{t}}+\mathbf{v}_{\mathrm{s}} \cdot \nabla \mathrm{M}=\frac{1}{\rho_{\mathrm{s}}} \nabla \cdot\left(\rho_{\mathrm{s}} \mathrm{D} \nabla \mathrm{M}\right) & \text { in } \Omega \\ -\rho_{\mathrm{s}} \mathrm{D} \nabla \mathrm{M} \cdot \mathbf{n}=\mathrm{J} & \text { on } \Gamma\end{cases}
$$

where $\Omega$ is the space domain occupied by the medium and $\Gamma$ its surface. In equation (1), coefficient $\mathrm{D}$ is defined in a solid-attached frame using the following equation (1 bis) (Ketelaars, 1992):

$$
\rho_{\mathrm{m}} \mathbf{v}_{\mathrm{m}}=\rho_{\mathrm{m}} \mathbf{v}_{\mathrm{s}}-\rho_{\mathrm{s}} \mathrm{D} \nabla \mathrm{M}
$$

The Lagrangian description has been applied to (1) in order to have a fixed computational domain (Kaasschieter, 1992; Ketelaars, 1992; Wigmans, 1994). Transformation rules (Chadwick, 1976) permit to switch from space co-ordinate system $\mathbf{x}(\mathrm{t})$ to solid-attached co-ordinates $\mathbf{z}=\mathbf{x}(0)$ and eliminate the solid movement term, by means of the deformation tensor $\left(\nabla_{\mathrm{z}} \mathbf{x}\right)^{\mathrm{C}}$. Deduced from the law of mass conservation, the following relation defines the shrinkage factor $\psi$ as inverse of the volume ratio:

$$
\operatorname{det}\left(\nabla_{\mathrm{z}} \mathbf{x}\right)^{\mathrm{C}}=\frac{\mathrm{dV}}{\mathrm{dV}_{0}}=\frac{\rho_{\mathrm{s}}^{0}}{\rho_{\mathrm{s}}}=\psi^{-1}
$$

At this stage an additional assumption is taken: deformation is only due to moisture removal, that is to say deformations due to mechanical stresses are smaller and therefore neglected. Hence deformations are purely volumetric and $\left(\nabla_{z} \mathbf{x}\right)^{\mathrm{C}}$ is a diagonal tensor. Furthermore, because of isotropy, $\left(\nabla_{z} \mathbf{x}\right)^{\mathrm{C}}$ has equal diagonal elements:

$$
\left(\nabla_{\mathrm{z}} \mathbf{x}\right)^{\mathrm{C}}=\psi^{-\frac{1}{3}} \mathbf{I}
$$

After transformation and applying (3), equations (1) reads (Hugget, 1999):

$$
\begin{cases}\left.\frac{\partial \mathrm{M}}{\partial \mathrm{t}}\right|_{\mathrm{z}}=\nabla_{\mathrm{z}} \cdot\left(\widetilde{\mathrm{D}} \nabla_{\mathrm{z}} \mathrm{M}\right) & \text { in } \Omega \\ -\rho_{\mathrm{s}}^{0} \widetilde{\mathrm{D}} \nabla_{\mathrm{z}} \mathrm{M} \cdot \mathbf{n}=\widetilde{\mathrm{J}} & \text { on } \Gamma\end{cases}
$$

where $\widetilde{\mathrm{D}}=\psi^{\frac{2}{3}} \mathrm{D}$ and $\widetilde{\mathrm{J}}=\psi^{-\frac{2}{3}} \mathrm{~J}$. Adding the initial condition, $\mathrm{M}(\mathrm{z}, \mathrm{t}=0)=\mathrm{M}^{0}, \mathrm{z} \in \Omega$, equations correspond to the mathematical formulation of the diffusion problem. 


\section{The stress model}

Let $\boldsymbol{\varepsilon}=\left(\nabla \mathbf{w}+\nabla_{\mathbf{w}}{ }^{\mathrm{C}}\right) / 2$ be the total strain tensor, function of the solid displacements $\mathbf{w}=\mathbf{x}(\mathrm{t})-\mathbf{z}$. With the assumption of a constant and uniform temperature, the total strain is reduced to the sum of the elastic strain and the shrinkage due to moisture (Kowalski, 1996):

$$
\boldsymbol{\varepsilon}=\boldsymbol{\varepsilon}^{\sigma}+\boldsymbol{\varepsilon}^{\mathrm{M}}
$$

The assumption of local isotropic shrinkage reads $\boldsymbol{\varepsilon}^{\mathrm{M}}=\varepsilon^{\mathrm{M}} \mathbf{I}$.

For an isotropic material, Hook's law describes the elastic strain:

$$
\boldsymbol{\varepsilon}^{\sigma}=\boldsymbol{\varepsilon}^{\mathrm{e}}=\frac{1+v}{\mathrm{E}} \boldsymbol{\sigma}-\frac{v}{\mathrm{E}} \operatorname{tr} \boldsymbol{\sigma} \mathrm{I}
$$

where $\mathrm{E}$ and $v$ are the moisture-dependent elastic properties of the mixture.

The balance of momentum for a static equilibrium reads:

$$
\nabla \sigma+\mathbf{F}=0
$$

By assumption, no external forces are applied to the clay body:

$$
\nabla \sigma=0
$$

On $\Gamma$, the boundary condition reads:

$$
\boldsymbol{\sigma} \cdot \mathbf{n}=0
$$

Inverting (6) and introducing (5), the momentum balance (7) and the boundary condition can be written in term of strains:

$$
\begin{cases}\nabla\left(\lambda \operatorname{tr} \boldsymbol{\varepsilon} \mathbf{I}+2 \mu \boldsymbol{\varepsilon}-3 \mathrm{~K} \varepsilon^{\mathrm{M}} \mathbf{I}\right)=0 & \text { in } \Omega \\ \left(\lambda \operatorname{tr} \boldsymbol{\varepsilon} \mathbf{I}+2 \mu \boldsymbol{\varepsilon}-3 \mathrm{~K} \varepsilon^{\mathrm{M}} \mathbf{I}\right) \cdot \mathbf{n}=0 & \text { on } \Gamma\end{cases}
$$

where $\lambda, \mu$ and $\mathrm{K}$ are properties of the mixture, functions of $\mathrm{E}$ and $v$. Equations (8) correspond to the mathematical formulation of the stress problem and its solution consists of the displacement $\mathbf{w}$ at the instant $t$. To solve this set of equations (8), the moisture content need to be known everywhere, so the diffusion problem should be solved at first.

\section{SIMULATION}

Based on the previous model, simulations of clay brick drying have been ran using the Finite Element Method, in order to study crack occurrence.

\section{Problem configuration}

We apply the mathematical model to a Kaolin clay brick submitted to drying with an initial moisture content of $0.4 \mathrm{~g} / \mathrm{g}$. Equations (4) and (8) are solved on a 2-dimensional mesh representing the quarter $\left(0.05 * 0.05 \mathrm{~cm}^{2}\right)$ of a cross-section of the clay brick, as shown on figure 1 . Hence the brick is assumed to have an infinite length. The mesh consists of $80 * 80$ rectangular elements.
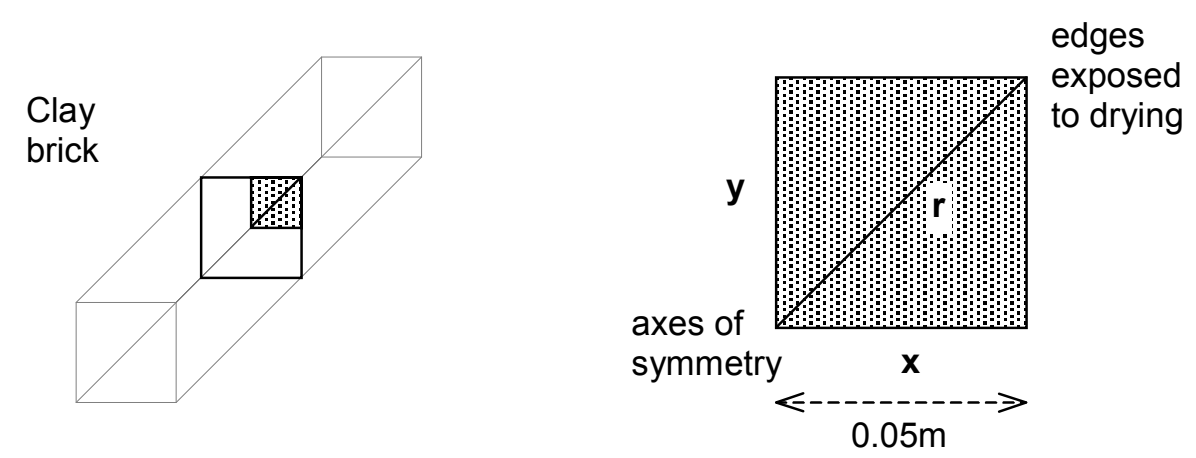

figure 1: Spatial configuration 


\section{Physical properties}

Moisture-dependent physical properties needed by the model were obtained experimentally by Ketelaars (1992).

The shrinkage factor $\psi(\mathrm{M})$ defined in equation (2), decreases linearly from $\mathrm{M}=0.4 \mathrm{~g} / \mathrm{g}$ to a critical moisture content $\mathrm{M}_{\mathrm{c}}=0.27 \mathrm{~g} / \mathrm{g}$ and then remains constant. Indeed clay drying consists in 2 phases: the shrinkage for $\mathrm{M}>\mathrm{M}_{\mathrm{c}}$ where solid particles slip into a more compact arrangement (Onoda, 1988), and the non-shrinking phase for $\mathrm{M}<\mathrm{M}_{\mathrm{c}}$ where solid density stays nearly constant.

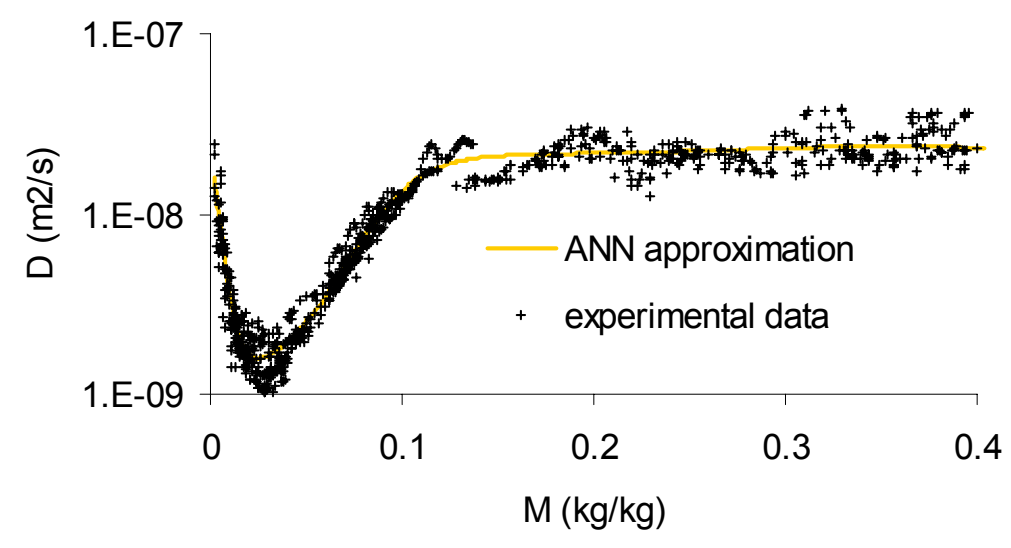

figure 2: Diffusion coefficient for Kaolin clay at $25^{\circ} \mathrm{C}$, obtained from 1-dim NMR profiles

The diffusion coefficient $\mathrm{D}$ for Kaolin clay has been determined experimentally from 1 dimensional drying experiments. Experimental data and the approximation of D using Artificial Neuronal Network (Hugget, 1999) are given figure 2. The prescribed drying flux $\mathrm{J}$ is derived from drying curves.

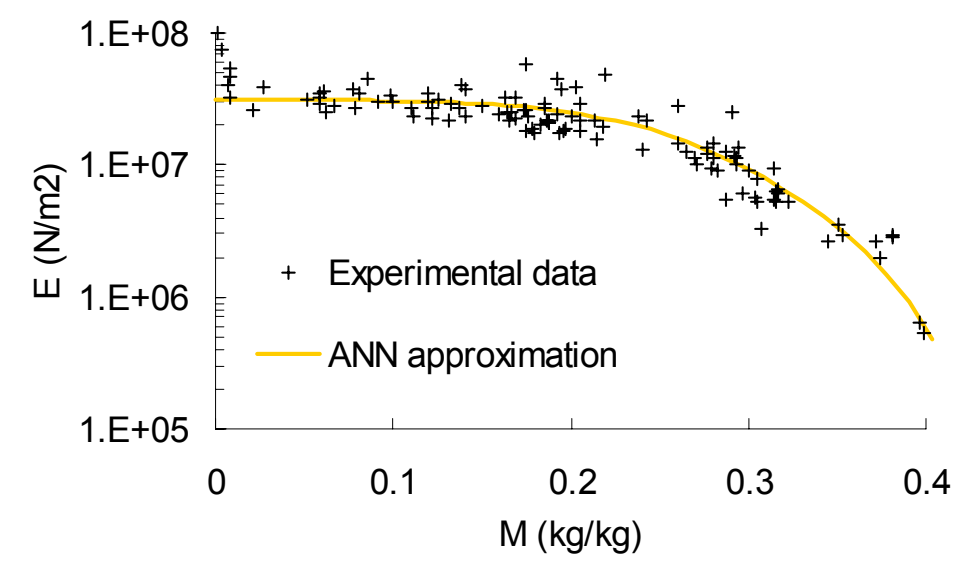

figure 3: Young's modulus for Kaolin clay (Ketelaars, 1992)

Compression tests at different moisture contents have led to an approximation of the Young's modulus E (figure 3). Poisson's coefficient is taken equal to 0.45 .

\section{RESULTS AND DISCUSSIONS}

Numerical results consist firstly of the moisture content evolution inside the 2 dimensional section, and secondly of displacements of the material points at different time-steps. From the displacements, total and elastic strains and thus stresses are derived, which permits to calculate a cracking criterion. On figures 4 to 10 , the legend refers to the drying hours. 


\section{Moisture and displacements}

Figure 4 shows moisture evolution along the diagonal of the slice. During the first drying stage moisture content decreases fast, especially in the corner where evaporation surface is important. We notice a slope break around $0.1 \mathrm{~kg} / \mathrm{kg}$, due to the evolution of D (see figure 2). After about $1 \mathrm{~h}$ of drying, a penetrating drying front appears inside the material, consequence of the increase of $\mathrm{D}$ at very low moisture contents (predominant vapor flow inside the pores).

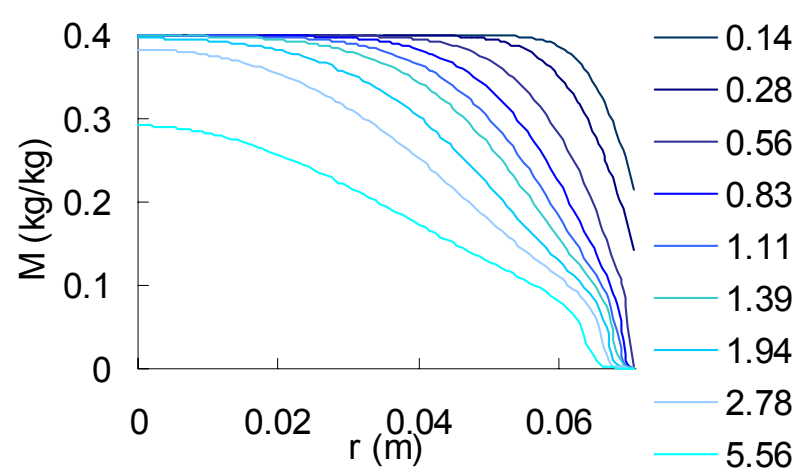

figure 4: Moisture evolution along r-direction (drying hours)

Figure 5 shows the displacement of the edge exposed to drying air. Because of its high drying rate, the corner undergoes a large strain at the beginning. It induces a deformation gradient from $y=0$ to $y=0.05$ and until $5 \mathrm{~h}$ of drying. After this time solid movement stops and the material returned to its initial shape. Indeed, below $\mathrm{M}_{\mathrm{c}}=0.27 \mathrm{~g} / \mathrm{g}$, volumetric strain ceases and, as a consequence of the linear elastic assumption, stress and elastic strain disappear: all solid particles have undergone the same strain.

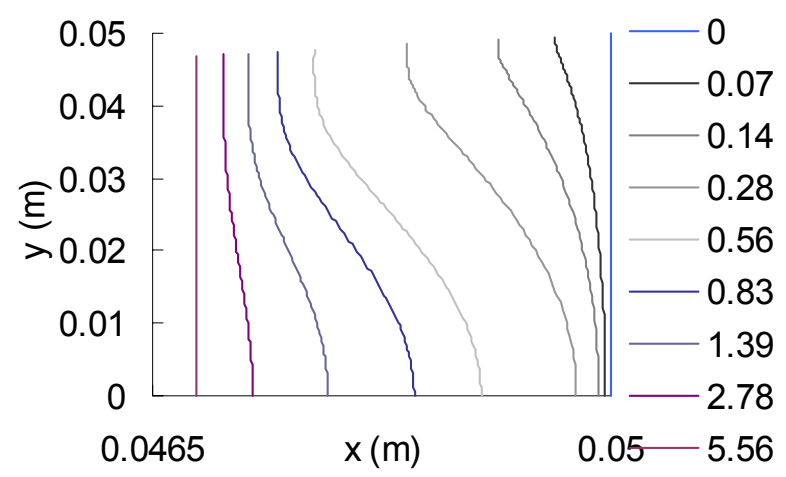

figure 5: Displacements of the edge $x(t=0)=0.05$ (at different drying hours)

\section{Stress analysis}

The conditions that lead to cracking can be determined by using a specific criterion. Since the crack appearance is independent of the hydrostatic pressure, we consider only the deviatoric part of the stress tensor, written down $\bar{\sigma}$. Then because of isotropic behaviour, the second invariant of $\bar{\sigma}$ is used. Hence, we will first focus on the following expression, called here the "stress criterion" and corresponding to the second invariant of the deviatoric part of the stress tensor:

$$
\sqrt{\frac{3}{2} \operatorname{tr}\left[\bar{\sigma}^{2}\right]}
$$

Figure 6 shows the evolution of the stress criterion along the diagonal $r$ of the section. Each profile shows a peak corresponding to the location where $M=M_{c}$, that is to say where strains have reached a maximum value (end of the shrinking phase). For each profile, above and below $\mathrm{M}=\mathrm{M}_{\mathrm{c}}$, the stress criterion is lower: 
Above $\mathrm{M}_{\mathrm{c}}$ (inner part), displacements and strains have not yet reached the maximum. Below $\mathrm{M}_{\mathrm{c}}$ (outer part) total strain becomes uniform which induces a decrease of stress.

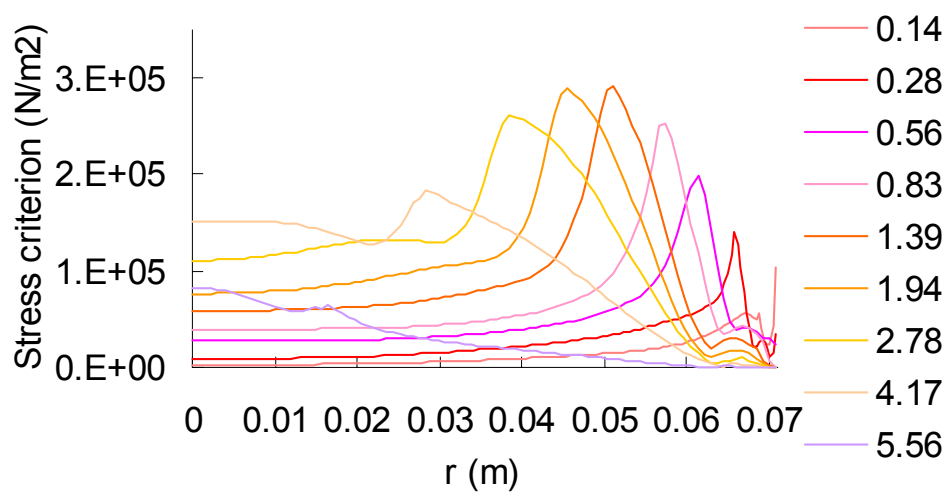

figure 6: Stress criterion along the r-direction (at different drying hours)

The Huber-von Mises yield criterion is proposed for problems in which frictions are not important (Shigley, 1989; Irudayaraj, 1993). This criterion states that in order to prevent cracking the following condition have to be satisfied:

$$
\mathrm{Y}-\sqrt{\frac{3}{2} \operatorname{tr}\left[\bar{\sigma}^{2}\right]}>0
$$

where $\mathrm{Y}(\mathrm{M})$ is the moisture-dependant yield stress, limit of the elastic behaviour of the material when submitted to a uniaxial tension. For Kaolin, Y has been determined experimentally by Ketelaars (figure7).

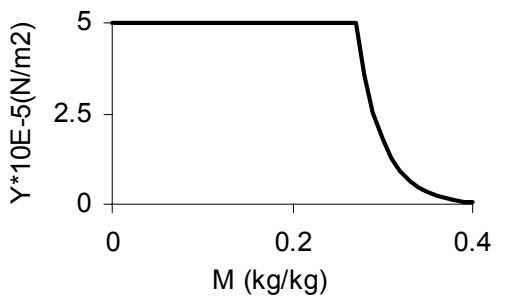

figure 7: Yield stress for Kaolin clay

Figures 8, 9 and 10 show the evolution of the cracking criterion along the $\mathrm{x}-, \mathrm{y}$ - and r-directions. Danger for cracking appears when the criterion is negative and increases with its absolute value. We notice from figures 8 and 9 that maximum danger occurs at $\mathrm{x}=0.05$ and $\mathrm{y}=0$, at time $0.56 \mathrm{~h}$. The risk remains important in this location during approximately the first 1.5 hour of drying. Danger is located especially on the edge (figure 8: $\mathrm{x}=0.05$ ) around axe of symmetry $\{\mathrm{y}=0\}$ of the brick (figure 9). As shown on figure 10, the criterion is higher inside the slice. After 1.5h of drying, risk for cracking is low everywhere.

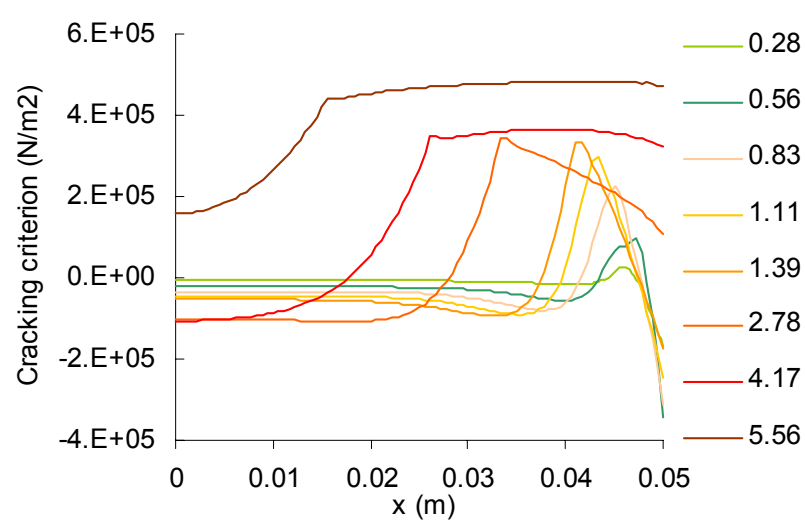

figure 8: Cracking criterion along the $\mathrm{x}$-direction

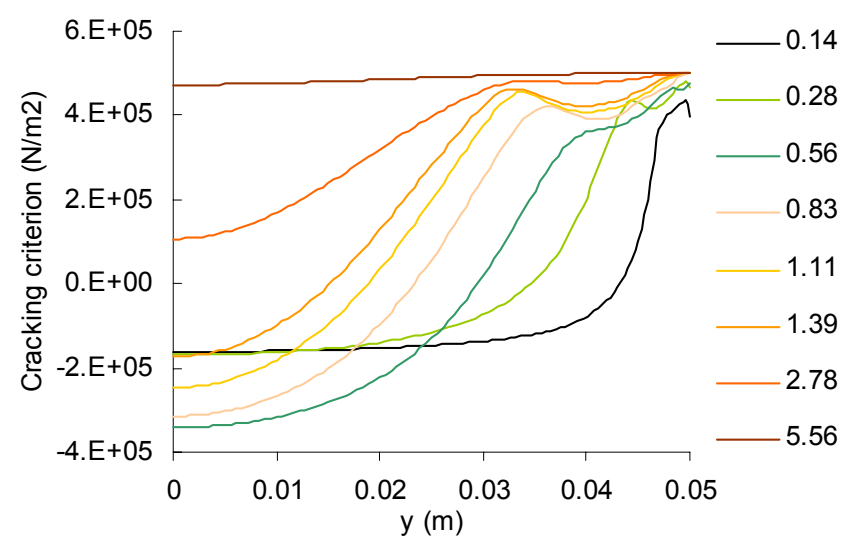

figure 9: Cracking criterion along the y-direction 

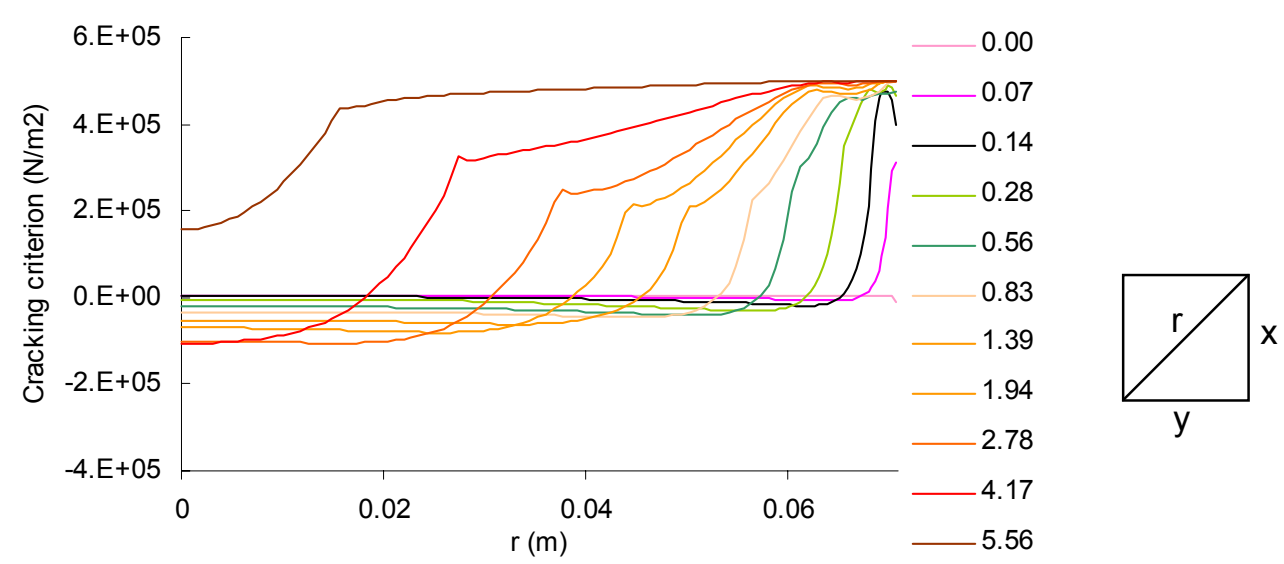

figure 10: Cracking criterion along the r-direction

The location of danger results from the drying rate difference between the corner and the middle of the edge, which induces important strain gradients along the edge (see figure 5). Thus the area around $\mathrm{x}=0.05$ and $y=0$ undergoes large tensile stress. Besides, at the beginning of the drying the yield stress is low because of the high moisture content. Combining this large tensile stress with the low yield stress, the danger for cracking becomes important.

\section{EXPERIMENTAL APPROACH}

The model and crack anticipation need to be validated by means of experiments. We present a result corresponding to the diffusion problem.

\section{2-dimensional moisture measurements using NMR-i}

A drying experiment using Nuclear Magnetic Resonance has been carried out on a Kaolin clay sample. With the objective to reproduce the corner of a brick, precautions are taken to ensure that moisture movement occurs in 2 dimensions. The sample has a right-angled triangle shape, as it can be noticed in figure 11, and both upper edges are identically exposed to a longitudinal drying airflow. The experimental data consist of the evolution with time of a 2D-moisture field.

Drying air specifications: $0 \% \mathrm{RH}, 1.7 \mathrm{~m} / \mathrm{s}$, room temperature $\left(19^{\circ} \mathrm{C}\right)$
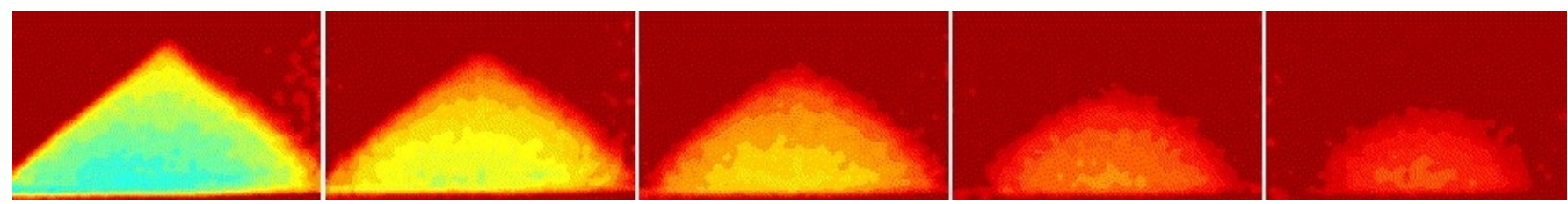

figure 11: NMR-imaging of clay drying (times: 0 - 1.5 - 3 - 5 - 10 hours), click here for animation

Figure $11^{*}$ shows the evolution of moisture qualitatively; light color corresponding to high moisture contents and dark to the absence of moisture. Both right and left sides of the sample have the same symmetric behavior. It appears clearly that the moisture in the corner decreases faster than along the edge. Since moisture content is above $0.27 \mathrm{~g} / \mathrm{g}$, it induces strain gradients between the corner and the middle of the edge, involving stresses. Differential strains on boundaries can be noticed on the first pictures of the animation.

\footnotetext{
${ }^{*}$ Click here to start the short experimental animation. You will need to download the free movie player Flashplayer
} 


\section{Diffusion coefficient from a 2-dimensional moisture evolution}

The diffusion coefficient defined in system (1) has been calculated from the results of the 2D experiment (figure 11), using a method based on the assumption that $\mathrm{D}$ depends only on moisture content (Augier, 2000). Flux and gradients are obtained along iso-moisture lines in order to calculate D. Results are showed on figure 12. The range of moisture content is reduced for 2 reasons. First the shrinkage is not taken into account for the calculations and second profiles measured at the beginning of the drying are too flat to get accurate gradients.

The coefficient used for the simulation (figure 2) is very near from this result in the moisture range [0$0.12 \mathrm{~g} / \mathrm{g}]$. We notice that $\mathrm{D}$ from the 2-dimensional drying is lower, what is explained by the difference of temperature between both experiments (about $5^{\circ} \mathrm{C}$ ). The agreement permits to validate the assumption of isotropy. Besides results from the 2-dimensional test are more accurate, because the method induces less averages than the 1-dimensional experiment, by taking in account boundary effects.

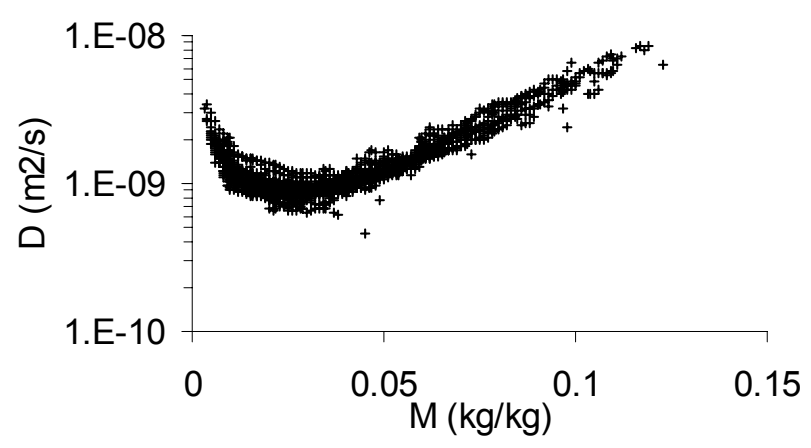

figure 12: Diffusion coefficient from 2D moisture profile evolution

\section{Comparison between model and experiment}

The parameters have been tuned to study how far the model can reproduce the experiment, and be validated by this way. Boundary drying fluxes have been found by integrating moisture profiles along the axis of symmetry of the sample, where it is assumed that no perpendicular gradients take place. Figure 13 shows the evolution of moisture content along the y-axis of the slice, plotted in a fixed unit. The experiment is well reproduced by the model, all along the drying stage. Low experimental points near the origin $(y=0)$ correspond to inaccuracies of the NMR signal, far from the center of the magnets. These errors cannot be seen clearly on figure 11 , because of some data averaging.

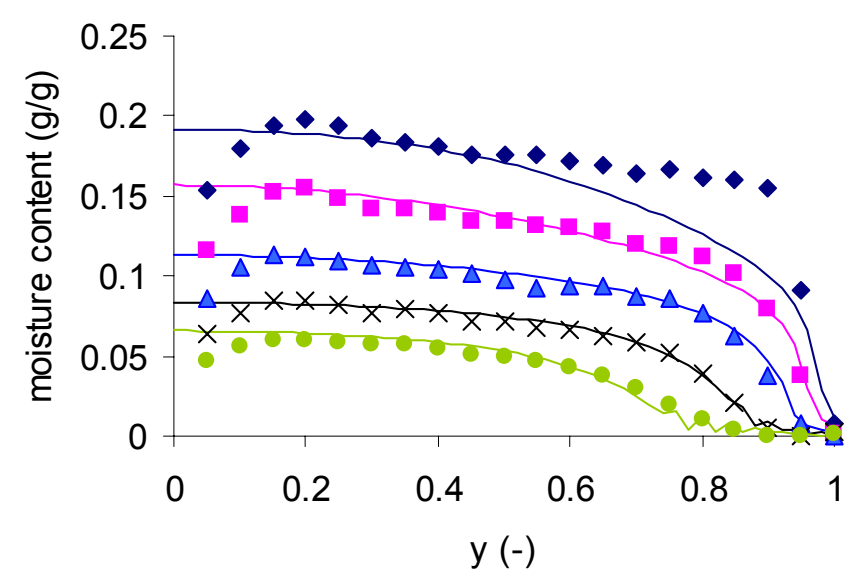

figure 13: Comparison between theory (lines) and experience (points) - profile times ( $\min ):$ 42, 98, 182, 322, 504. 


\section{CONCLUSIONS}

From a mathematical model including moisture transport in porous medium and balance of momentum for static equilibrium, a numerical solution is obtained by means of the finite element method. Both moisture content evolution and solid displacements are calculated all over a cross-section of a Kaolin clay brick. Since the material is assumed to be perfectly elastic, strains and stresses are obtained and the Huber-von Mises cracking criterion is analyzed. Maximum risk for cracking occurs at the surface around the axe of symmetry of the brick and, according to the drying conditions applied, after $0.56 \mathrm{~h}$ of drying.

A validation of the diffusion model is given, based on the calculation of the diffusion coefficient from a 2-dimensional moisture evolution measured by Nuclear Magnetic Resonance. A good agreement has been found between the coefficient from the 1-dimensional drying tests and from the 2-dimensional NMR experiment, for the non-shrinking phase. We aim to generalize these 2-dimensional calculations for the whole drying process and to increase the accuracy of the method, which could be applied favorably to products with a more complex shape.

The experimental study of crack appearance is now in progress, in order to estimate the stress model and the use of the cracking criterion. For the improvement of the stress model, more rheological properties, such as viscosity, need to be known all over the moisture range. This supposes to realize mechanical tests before simulating the viscous behavior.

\section{NOTATION}

$\begin{array}{lll}\text { D } & \text { diffusion coefficient } & \mathrm{m} / \mathrm{s} \\ \text { E } & \text { Young modulus } & \mathrm{N} / \mathrm{m}^{2} \\ \mathbf{F} & \text { external stresses } & \mathrm{N} / \mathrm{m}^{2} \\ \text { J } & \text { prescribed drying flux } & \mathrm{kg} / \mathrm{m}^{2} . \mathrm{s} \\ \text { K } & \text { bulk modulus } & \mathrm{N} / \mathrm{m}^{2} \\ \text { M } & \text { dry basis moisture content } & \mathrm{kg} / \mathrm{kg} \\ \mathbf{n} & \text { surface normal vector } & - \\ \mathbf{v} & \text { velocity } & \mathrm{m} / \mathrm{s} \\ \mathbf{w} & \text { displacement vector } & \mathrm{m} \\ \mathbf{X} & \text { spatial position } & \mathrm{m} \\ \mathbf{Z} & \text { solid-attached position } & \mathrm{m} \\ & & \\ G r e e k & S y m b o l s & \\ \boldsymbol{\varepsilon} & \text { total strain tensor } & - \\ \boldsymbol{\varepsilon}^{\mathrm{e}} & \text { elastic strain tensor } & - \\ \boldsymbol{\varepsilon}^{\mathrm{M}} & \text { strain tensor due to moisture removal } & - \\ \boldsymbol{\varepsilon}^{\sigma} & \text { mechanical strain tensor } & - \\ \lambda, \mu & \text { Lamé coefficients } & \mathrm{N} / \mathrm{m}^{2} \\ \nu & \text { Poisson ratio } & - \\ \rho & \text { apparent bulk mass density } & \mathrm{kg} / \mathrm{m}^{3} \\ \sigma & \text { stress tensor } & \mathrm{N} / \mathrm{m}^{2} \\ \psi & \text { shrinkage factor } & -\end{array}$

\section{LITERATURE}

Augier, F., 2000, Diffusion Coefficient from a 2 dimensional moisture field, Internal Report of SST, Eindhoven University of Technology, The Nederlands

Chadwick, P., 1976, Continuum Mechanics, George Allen \& Unwin LTD, London 
Coumans, W.J., 1987, Power law diffusion in drying processes, Ph.D. Thesis, Eindhoven University of Technology, The Nederlands

Hugget, A., Coumans, W.J., Kaasschieter, E.F., 1999, Drying-induced Stress and Deformation for Clays, Reports on applied and numerical analysis, RANA99-30, Eindhoven University of Technology, 28p.

Irudayaraj, J., Haghighi, K. and Stroshine, R., 1993, Stress analysis of viscoelastic materials during drying, Drying Technology, 11(5), pp. 901-959

Kaasschieter, E.F., 1992, Een eindige elementmethode voor het modelleren van het drogen van kleivormlingen, Eindhoven University of Technology, The Nederlands

Ketelaars, A.A.J., 1992, Drying deformable media: Kinetics, Shrinkage and Stresses, Ph.D. Thesis, Eindhoven University of Technology, The Nederlands

Kowalski, S.J., 1996, Drying Processes involving permanent Deformations of dried Materials, Int.J.EngngSci., 34(13), pp.1491-1506

Kroes, B., 1999, The Influence of Material Properties on Drying Kinetics, Ph.D. Thesis, Eindhoven University of Technology, The Nederlands

Onoda, G., Liniger, E.G. and Janney, M.A., 1988, Dilatancy and Plasticity in Ceramic Particulate Bodies, Ceram. Trans. 1 (Ceramic Powder Sci.II), Eds Messing, Fuller and Hausner, pp.611-623

Wigmans, A., 1994, A model for Drying Process of Clay, Master's Thesis, Eindhoven University of Technology, The Nederlands 